\title{
TRIM47 promotes malignant progression of renal cell carcinoma by degrading P53 through ubiquitination
}

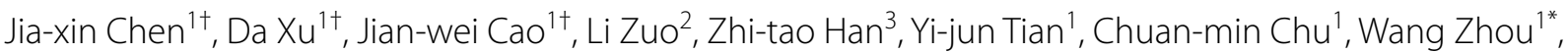 \\ Xiu-wu Pan ${ }^{1 *}$ and Xin-gang Cui ${ }^{*}$ (D)
}

\begin{abstract}
Background: Renal cell carcinoma $(\mathrm{RCC})$ is one of the most common malignant tumors originating from the renal parenchymal urinary epithelial system. Tripartite motif 47 (TRIM47) is a member of the TRIM family proteins, which has E3 ligase activity and has been demonstrated to be involved in the occurrence and prognosis of many tumors. The main purpose of this study is to explore the role and potential mechanism of TRIM47 in promoting malignant biological behavior of RCC.
\end{abstract}

Materials and methods: TRIM47 mRNA and protein levels in human renal cancer and paired normal adjacent tissues were detected by qRT-PCR and Western blot. The effects of TRIM47 knockdown and overexpression in renal cell carcinoma cells on cell proliferation, invasion and xenograft tumor growth in nude mice were analyzed. The molecular mechanism was explored by mass spectrometric exploration,Western blot and immunoprecipitation assays.

Results: TRIM47 promoted RCC cell proliferation in vitro and in vivo as an oncogene. Mechanistically, TRIM47 exerted an E3 ligase activity by interacting with P53 protein to increase its ubiquitination and degradation, which further promoted the malignant biological behavior of RCC.

Conclusions: Our study demonstrated that the TRIM47-P53 axis played a functional role in RCC progression and suggested a potential therapeutic target for RCC.

Keywords: Renal cell carcinoma, E3 ubiquitin ligase, Proliferation, P53, TRIM47

\section{Background}

Renal cell carcinoma (RCC) is a common malignant tumor of the urogenital system, the incidence of which is only next to that of prostate cancer and bladder cancer, accounting for $2.2 \%$ of adult malignant tumors [1]. At present, radical surgery remains the mainstay of

\footnotetext{
*Correspondence: brilliant212@163.com; panxiuwu@smmu.edu.cn; cuixingang@smmu.edu.cn

${ }^{\dagger}$ Jia-xin Chen, Da Xu, Jian-wei Cao and Li Zuo contributed equally to this work

1 Department of Urology, Third Affiliated Hospital of the Second Military Medical University, 200433, China, Shanghai

Full list of author information is available at the end of the article
}

treatment for patients with early-stage RCC [2]. However, due to the asymptomatic or covert symptoms of kidney cancer in the early stage and the lack of awareness of cancer screening, patients often missed the early diagnosis and treatment, and at the time of diagnosis, local progression had occurred, or the condition was already in the advanced clinical stage [3].

The advent of targeted drugs as adjuvant therapy and their promising therapeutic efficacy have brought new hope for patients with high-risk and locally advanced RCC patients. Tyrosine kinase inhibitors (TKIs), such as sunitinib, axitinib, perzopanib and sorafenib, are the most commonly used molecular targeted drugs for

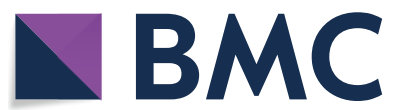

(c) The Author(s) 2021. This article is licensed under a Creative Commons Attribution 4.0 International License, which permits use, sharing, adaptation, distribution and reproduction in any medium or format, as long as you give appropriate credit to the original author(s) and the source, provide a link to the Creative Commons licence, and indicate if changes were made. The images or other third party material in this article are included in the article's Creative Commons licence, unless indicated otherwise in a credit line to the material. If material is not included in the article's Creative Commons licence and your intended use is not permitted by statutory regulation or exceeds the permitted use, you will need to obtain permission directly from the copyright holder. To view a copy of this licence, visit http://creativeco mmons.org/licenses/by/4.0/. The Creative Commons Public Domain Dedication waiver (http://creativecommons.org/publicdomain/ zero/1.0/) applies to the data made available in this article, unless otherwise stated in a credit line to the data. 
kidney cancer. They are vascular endothelial growth factor receptors (VEGFRs) that reduce tumor cell growth by targeting the tumor angiogenesis pathway $[4,5]$. However, most patients using TKIs may develop drug resistance and experience tumor progression early in treatment. In addition, patients with advanced kidney cancer are not sufficiently sensitive to sunitinib $[6,7]$. It is therefore necessary to further explore the complex mechanism underlying the development and progression of RCC.

Tripartite motif-47 (TRIM47) is a member of the TRIM protein family. The most important structure in this motif is a really interesting new gene (RING) domain. Proteins with a RING domain often play the role of E3 ligase activity [8-10]. TRIM47 has a RING structure and is reportedly defined as E3 ligase [11].TRIM47 was first determined to be overexpressed in astroglioma, which is located at 17q24-25. This region can be found or amplified in many other types of tumors [12], and high levels of TRIM47 expression have been reported. It is a strong prognostic factor for prostate cancer [13]. TRIM47 overexpression could promote the occurrence of non-small cell lung cancer (NSCLC) [14], and TRIM47 could play the role of E3 ligase by interacting with SMAD4 to promote SMAD4 ubiquitination and degradation, which further increased the level of $\mathrm{CC}$ motif chemokine ligand 15 (CCL15) and CC motif chemokine receptor 1 (CCR1), ultimately leading to poor prognosis of colorectal cancer [15]. Therefore, we believe that TRIM47 is an important proto-oncogene involved in the development and progression of various malignant tumors. However, the expression and biological function of TRIM47 in RCC have not been reported.

The purpose of this study was to further determine the role of TRIM47 in the development and progression of RCC by immunohistochemical staining of RCC specimens, construction of TRIM47-overexpression plasmids, lentiviral shTRIM47 knockdown cell line, and Crispr/ cas9 technology to construct knockout methods such as TRIM47 stably transformed strains, animal experiments, mass spectrometry detection, bimolecular fluorescent complimentary experiments and co-immunoprecipitation experiments, in an attempt to gain deeper insights into the molecular biological function and mechanism of TRIM47 in RCC.

\section{Materials and methods}

\section{Patients and tissue samples}

Clinical specimens of kidney cancer and paired adjacent tissues were collected from 6 patients who received surgical treatment between October 2017 and February 2018 at the Department of Urology of the Oriental Hepatobiliary Surgery Hospital affiliated to the Naval
Medical University (Shanghai, China). They included 4 pairs of clear cell renal cell carcinoma (CCRCC), one pair of papillary renal cell carcinoma (PRCC) and one pair of chromophobe renal cell carcinoma (CRCC). The postoperative clinical specimens of 125 patients constituted paired RCC and paired normal adjacent tissues. Clinicopathological variables including age at age, gender, tumor size, pathological types and tumor stages were analyzed (Table 1).

\section{Cell culture}

Both RCC cell lines (A498, 786-O, ACHN, 769-P, Caki-1 and OS-RC-2) and normal renal cell HK-2 (ATCC, USA) were routinely maintained in RPMI-1640 (Gibco, USA), DMEM (Gibco) and McCoy's 5A supplemented with $10 \%$ FBS (Gibco), $100 \mathrm{U} / \mathrm{mL}$ penicillin sodium and $100 \mathrm{mg} /$ $\mathrm{mL}$ streptomycin in a humid atmosphere with $5 \% \mathrm{CO}_{2}$ at $37^{\circ} \mathrm{C}$.

\section{Immunohistochemistry (IHC)}

After dewaxing, hydration and antigen repair, the renal cell carcinoma tissue chip was added dropwise with diluted rabbit anti-TRIM47 antibody (1:500, ab155549, Abcam, USA) and incubated overnight. The diluted antirabbit HRP and horseradish enzyme-labeled streptavidin working solution were added dropwise to the tissue chip the next day. The tissue chip was then stained with

Table 1 Relationship between TRIM47 protein expression level and clinicopathological factors in RCC patients

\begin{tabular}{|c|c|c|c|c|}
\hline \multirow{2}{*}{$\begin{array}{l}\text { Clinicopathological } \\
\text { variables }\end{array}$} & \multirow{2}{*}{$\begin{array}{l}\text { No. } \\
\text { Total } 125\end{array}$} & \multicolumn{2}{|c|}{ TRIM47 expression } & \multirow[t]{2}{*}{ P value } \\
\hline & & Low & High & \\
\hline Gender (\%) & & & & 0.828 \\
\hline Male & 92 & $64(74.4)$ & $28(71.8)$ & \\
\hline Female & 33 & $22(25.6)$ & $11(28.2)$ & \\
\hline Age (\%) & & & & 0.845 \\
\hline$<60$ & 73 & $51(59.3)$ & $22(56.4)$ & \\
\hline$\geq 60$ & 52 & $35(40.7)$ & $17(43.6)$ & \\
\hline TNM (\%) & & & & $<0.01^{* *}$ \\
\hline 1 & 83 & $70(81.4)$ & $13(33.3)$ & \\
\hline 2 & 20 & $14(16.3)$ & $6(15.4)$ & \\
\hline 3 & 22 & $2(2.3)$ & $20(51.3)$ & \\
\hline Fuhrman (\%) & & & & $<0.01^{* *}$ \\
\hline 1 & 20 & $19(22.1)$ & $1(2.6)$ & \\
\hline 2 & 68 & $53(61.6)$ & $15(38.5)$ & \\
\hline 3 & 23 & $11(12.8)$ & $12(30.8)$ & \\
\hline 4 & 14 & $3(3.5)$ & $11(28.2)$ & \\
\hline OS (\%) & & & & $<0.01^{* *}$ \\
\hline+ & 104 & 79 (91.9) & $25(64.1)$ & \\
\hline- & 21 & $7(8.1)$ & $14(35.9)$ & \\
\hline
\end{tabular}


DAB chromogen and counterstained with hematoxylin, dehydrated and observed and counted under an optical microscope. The results of histochemical staining and the evaluation criteria of the staining intensity were scored as 0 for negative, 1 for weakly positive, 2 for moderately positive, and 3 for strongly positive. The evaluation criteria for positive cell frequency were as follows: $<5 \%$, 0 point; $5-25 \%, 1$ point; $26-50 \%, 2$ points; $51-75 \%, 3$ points; and $>75 \%, 4$ points.

\section{TIRM47 overexpression plasmids and RNA interference}

The coding sequence of TRIM47 was synthesized by TSINGKE Biological Technology (Shanghai, China), and then inserted into pcDNA3.1(+) using the QuickFusion cloning kit (Biotool, USA).The shRNA sequence of TRIM47 was CAAGAAGTCCTGCATATCCGT. After $293 \mathrm{~T}$ cells were infected with the lentivirus packaging plasmid, and 769-P and A498 cells were infected with the virus for $48 \mathrm{~h}$, they were observed under a fluorescence microscope.

\section{Quantitative real-time PCR (qRT-PCR)}

TRIZOL (Invitrogen, USA) was used to extract the total RNA, and then reverse transcribed into cDNA. Finally, SYBR Premix Ex TaqTM II (Takara, Japan) was used to quantify gene transcripts on the $7900 \mathrm{HT}$ fast RT-PCR system (Life Technologies Corporation, USA) and standardized with GAPDH.Primer sequence: GAPDH-F: GTC TCCTCTGACTTCAACAGCG; GAPDH-R: ACCACC CTGTTGCTGTAGCCAA; TRIM47-qF: ACGGCAGTG GACCCTTCAG; TRIM47-qR: CCAGGCAGGCGA GACAGAA.

\section{Western blot analysis}

Total protein was first extracted using SDS-PAGE and then transferred to a PVDF membrane (Thermo, USA). Afterwards, the PVDF membrane was incubated with 1:1000 dilution of antibody: TRIM47 (ab155549, Abcam, USA), BAP1 (ab199396, Abcam, USA), P21 (ab188224, Abcam, USA), P53 (ab131442, Abcam, USA), SRC (ab47405, Abcam, USA), MET (ab51067, Abcam, USA), and c-Myc (ab39688,Abcam, USA). The membrane was washed and incubated with a 1:2000 dilution of horseradish peroxidase-conjugated goat anti-rabbit (Santa Cruz, USA). SuperSignalMT West Puico PLUS (Termo Scientific, USA) was used to develop the blot, using b-actin (ab8226, Abcam, USA) as the loading control. All experiments were performed in triplicate.

\section{CCK8 assays}

After digestion, counting and centrifugation, overexpression cells or knockdown cells were seeded into 96-well plates with 5000 cells per well, cultured for $24 \mathrm{~h}$, and finally evaluated using the Cell Counting Kit 8 (Biotool, USA). The absorbance at $450 \mathrm{~nm}$ was measured using an ELx800 plate reader (BioTek Instruments Inc., USA).

\section{Transwell invasion assay}

Matrigel was placed on ice in advance to hydrate overnight, and then diluted with serum-free medium at a 1:8 ratio, followed by addition of $50 \mu \mathrm{l}$ matrigel to the bottom of the transwell chamber. Cells were digested, counted and then inoculated onto the transwell chamber. The total amount of cells per well was $0.4 \times 10^{5} / \mathrm{mL}$. The bottom of the chamber is 1640 medium containing $15 \% \mathrm{FBS}$, which is placed at $\left(37{ }^{\circ} \mathrm{C}, 5 \% \mathrm{CO}_{2}\right)$ for $48 \mathrm{~h}$. Cells were fixed in $10 \%$ methanol solution for $30 \mathrm{~min}$, stained with $0.1 \%$ crystal violet for $20 \mathrm{~min}$, and washed in tap water until the background was clear. Five fields were randomly selected under the light microscope, and the number of membrane-passing cells was counted.

\section{Crispr/Cas9 technology}

Crispr/Cas9 system was used to construct the knockoutTRIM47-769P cell. After constructing the sgRNA-cas9 expression vector and transfecting the 769P cell line, the stable transgenic strain was constructed through the lentiviral packaging system and selected by puromycin. The sgRNA sequence of TRIM47 is CGCCTGCCTGGGCGC GCTCTGG.

\section{In vivo xenograft assay}

The experimental procedures were approved by the Second Military Medical University. Male BALB/c-nu mice (4-5 weeks old, 18-20 g) were purchased from Shanghai Laboratory Animal Company (SLAC, Shanghai). Sixteen nude mice were equally randomized into two groups: WT-769P group and KO-TRIM47-769P group. After adjustment of the concentration to $1 \times 10^{7} / \mathrm{mL}$, each mouse was injected with $0.2 \mathrm{~mL}$ cells (about 2 million cells) into the upper right thigh and observed for 3 weeks.

\section{Co-immunoprecipitation}

The TRIM47 sequence was inserted into the Flag-tagged vector, and the P53 sequence was inserted into the Histagged vector to form two recombinant plasmids. The recombinant plasmid carrying the target gene and the virus packaging plasmid were co-transfected into 293T cells and then infected 769-P cells. The corresponding stable transfected cells were screened using puromycin. The His-P53 overexpression stable transfected cells and His-P53 + Flag-TRIM47 overexpression stable transfected cells were obtained. After the extraction of the immunoprecipitated protein, the antibody was combined 
with protein $\mathrm{A} / \mathrm{G}$ agarose, and then the bound antibody was crosslinked, followed by pre-clearing of the protein sample, antigen immunoprecipitation and antigen elution before Western Blot detection was performed.

\section{Data analysis}

Statistical data are expressed as means \pm standard deviation (SD). All data were statistically analyzed and processed using SPSS (IBM SPSS Statistics 22.0) software. The experiment was repeated three times and then analyzed statistically.

\section{Results}

TRIM47 gene overexpression is associated with poor survival in RCC

The results of Western Blot detection of the fresh RCC and matched normal adjacent tissues indicated that the expression level of TRIM47 protein in RCC tissues was significantly higher than that in adjacent tissues (Fig. 1a). IHC staining of the tissue chips in the 125 cases of RCC and normal adjacent tissues showed that the staining degree of TRIM47 antibody in RCC tissues was significantly deeper than that in adjacent tissues, and the number of positive cells was higher (Fig. 1c) $(\mathrm{P}<0.001)$. qRT-PCR detection of the fresh RCC and paired adjacent tissues demonstrated that the mRNA expression level of TRIM47 in RCC tissues was up-regulated compared with that in normal adjacent tissues (Fig. 1b) $(\mathrm{P}<0.01)$. TCGA database analysis showed that RCC patients with high expression of TRIM47 in RCC had shorter overall survival (OS) and poorer prognosis than those with low expression of TRIM47 (Fig. 1d, f). Moreover, the same overall survival(OS) difference was displayed by the Kaplan-Meier survival analysis of RCC patients from the Department of Urology, Third Affiliated Hospital of the Second Military Medical University (Fig. 1e).

\section{Intervention of TRIM47 expression significantly affects RCC cell proliferation and invasion}

To clarify the biological effect of TRIM47 in RCC, we firstly detected the expression of TRIM47 in 6 different RCC cell lines and normal renal cell HK2 by using Western blot and qRT-PCR, and the results showed that $769 \mathrm{P}$ and A498 cells highly expressed TRIM47 while 786-O and ACHN cells lowly expressed TRIM47 (Fig. 2a, b).Then the overexpression-TRIM47 cells (786-O,ACHN) and knockdown-TRIM47 cells(769P,A498) were constructed and observed by fluorescence microscope and Western blot (Fig. 2c, d). The effect of TRIM47 expression level on RCC cell proliferation and invasion was evaluated in the CCK-8 cell proliferation experiment and transwell cell invasion experiment. The result showed that the RCC cell proliferation and invasion abilities were significantly enhanced in OE-TRIM47 group, while the RCC cell proliferation and invasion abilities were significantly reduced in KD-TRIM47 group, showing a statistically significant difference (Fig. $3 \mathrm{a}, \mathrm{b})(\mathrm{P}<0.01)$.

Mass spectrometric exploration of TRIM47 as the molecular mechanism underlying the malignant biological behavior of RCC cells

To understand the molecular mechanism of TRIM47 promoting the malignant biological behavior of RCC, we used Crispr/Cas9 technology to construct the knockoutTRIM47-769P cell. Then the sequencing result showed that mutations occurred near the sgRNA of TRIM47 target site, and Western blot was used to further verify the knockout efficiency (Fig. 4a). Differentially expressed proteins between KO-TRIM47-769P group and WT769P group were analyzed by mass spectrometry (MS) (Fig. 4b-d). According to the MS results, important tumor suppressor proteins (BAP1, P21 and P53) and cancer-promoting proteins (SRC, MET and c-Myc) were detected by Western blot to observe their changes in OETRIM47 and KO-TRIM47 groups. The results showed that the tumor suppressor proteins were down-regulated while the tumor-promoting proteins were up-regulated in OE-TRIM47 group, and vise versa in KO-TRIM47 group (Fig. 5a).

\section{Detection of the interaction between TRIM47 and P53 in 769-P RCC cell line by co-immunoprecipitation assay} Given that TRIM47 plays the E3 ubiquitin ligase role, we detected the relationship between TRIM47 and P53 in RCC. Firstly, we used the 769P cell line to construct the stable His-P53 overexpression stable transfected cells and the His-P53 + Flag-TRIM47 overexpression stable transfected cells and then detected by Western blot. The ubiquitin exposed a large number of clear bands, indicating that the antibody for detecting ubiquitin protein is good, and a large number of proteins in the cell are ubiquitinated (Fig. $5 \mathrm{~b}$ left panel). Furtherly, the protein interaction between TRIM47 and P53 in RCC 769-P cell line was detected by coimmunoprecipitation assay, and the result showed that the His antibody can pull out His-P53 itself, but the IgG does not pull out the band, indicating that the IP experiment was successful and there was no false positive. His antibody can pull out the ubiquitin of cells overexpressing His-P53, indicating that P53 is partially bound to ubiquitinated protein, and P53 is partially ubiquitinated. However, ubiquitin was obviously pulled out when His-P53 was overexpressed in Flag-TRIM47, indicating that His-P53 binds to ubiquitinated proteins more under the action of 


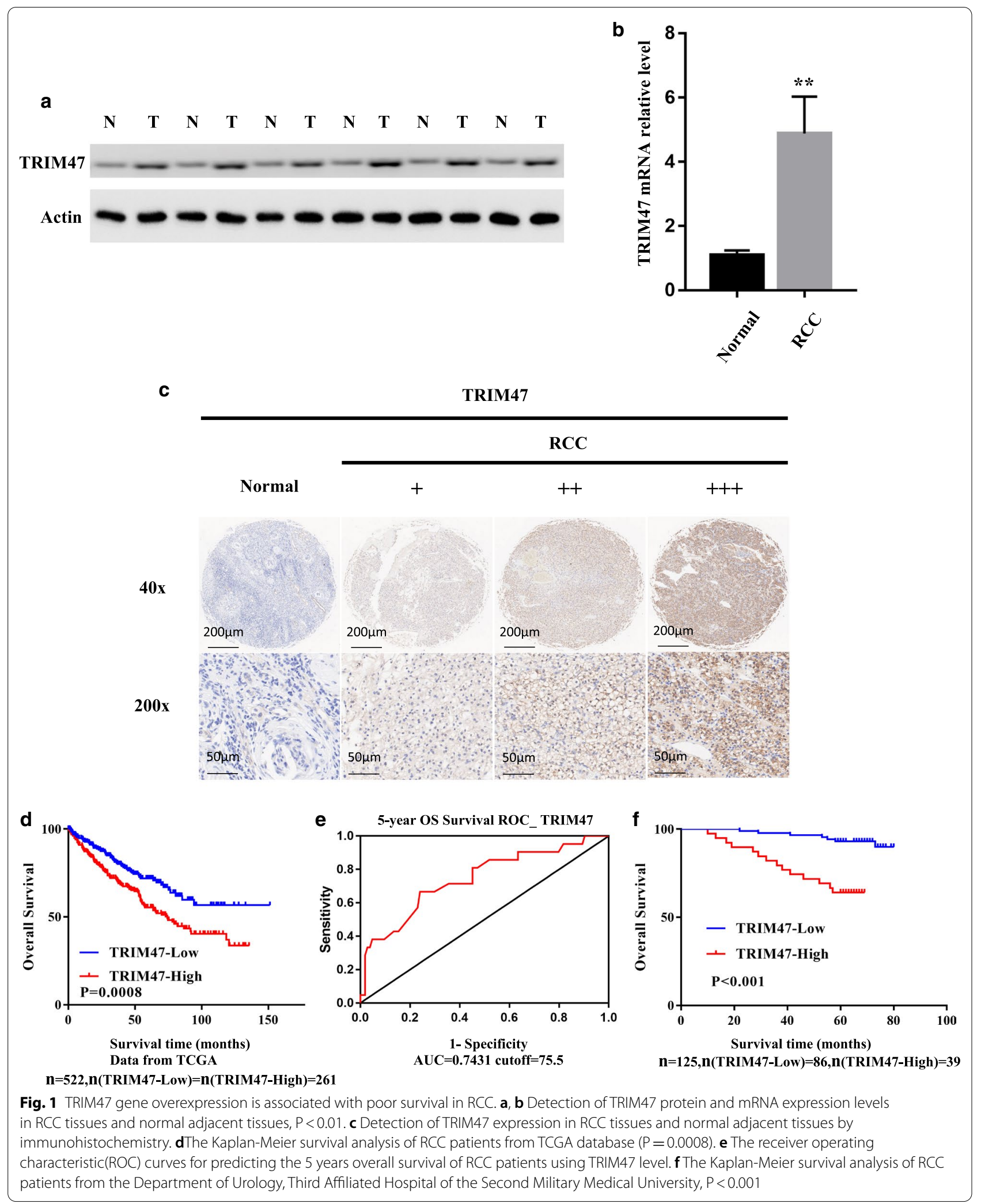



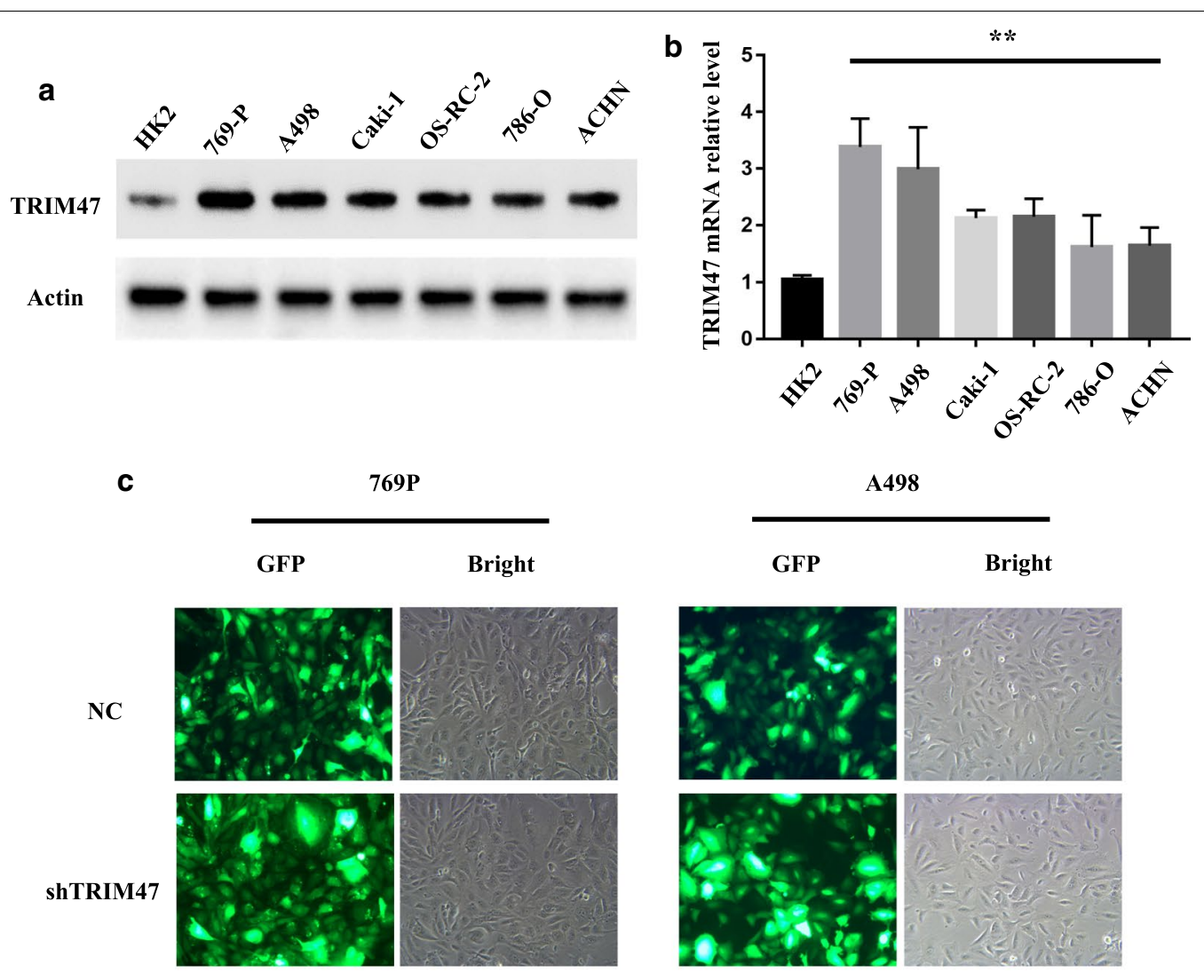

$\mathbf{d}$

786-O
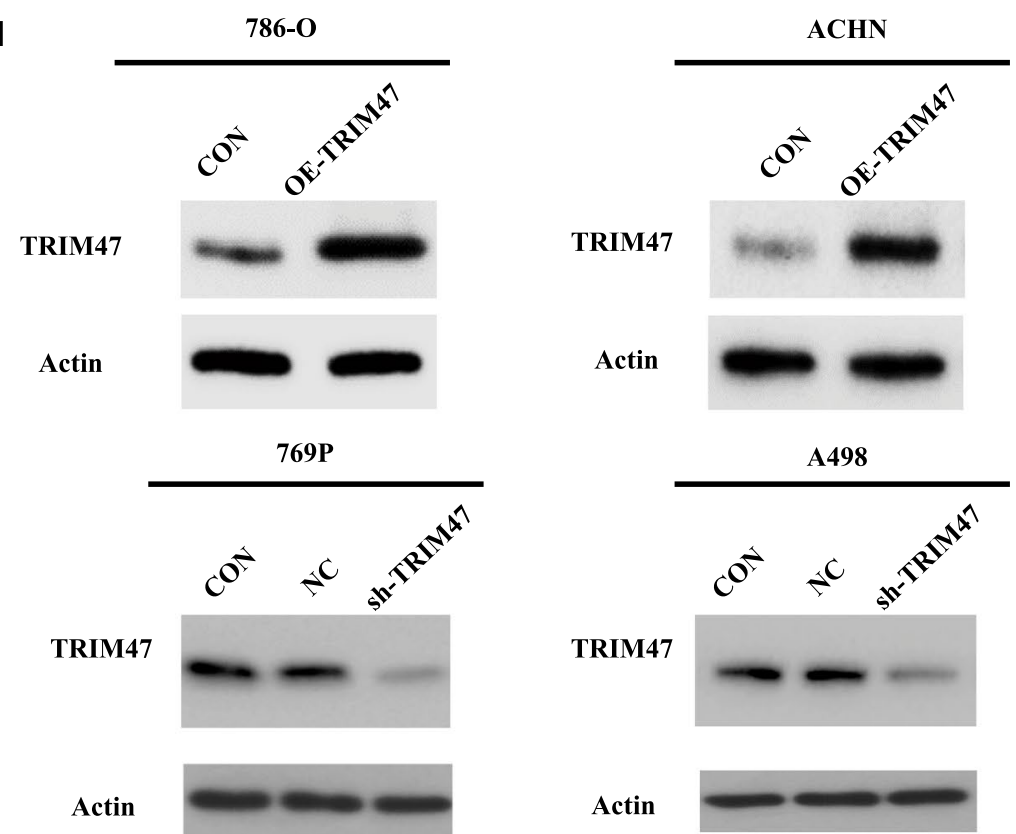

Fig. 2 The expression level of TRIM47 in RCC cell lines and normal renal cell HK2 and the construction of RCC cell lines that interfere with TRIM47. a Detection of TRIM47 protein expression level in 6 different RCC cell lines and normal renal cell HK2 by Western Blot. b Detection of TRIM47 mRNA expression level in 6 different RCC cell lines and normal renal cell HK2 by real time PCR. c Lentivirus-mediated shTRIM47 was used to infect 769-P and A498 cells. $\mathbf{d}$ Western Blot was used to verify the constructed TRIM47 overexpression and TRIM47 knockdown renal cancer cell lines 


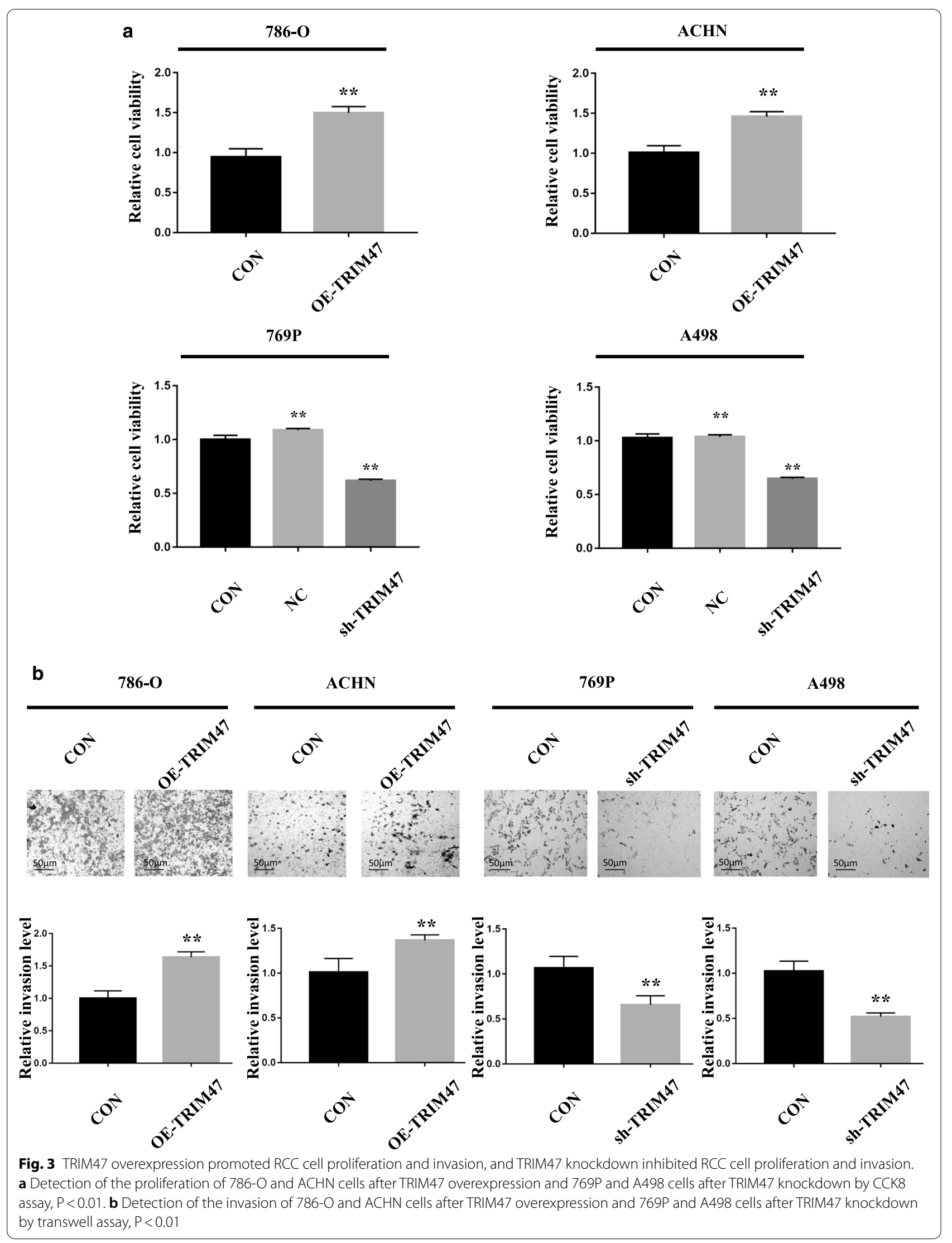


WT TGCCCTGCGGCCACAACT TCTGTCTCGCCTGCCTGGGCGCGCTCTGGCCGCATCGTGGC Ko-TRIM47 T G C C C T G C G G C C A C A A C T T C T G T C T C G C C T G C C T G G G C G C G C T C T G G C C GG T G G G C C G G

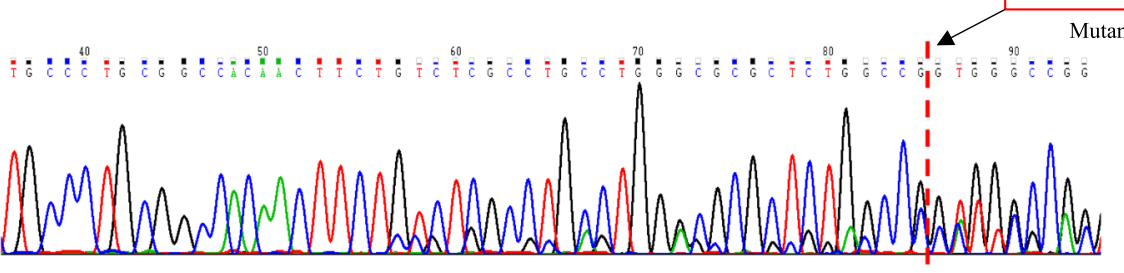

WT KO

TRIM47

Actin

b

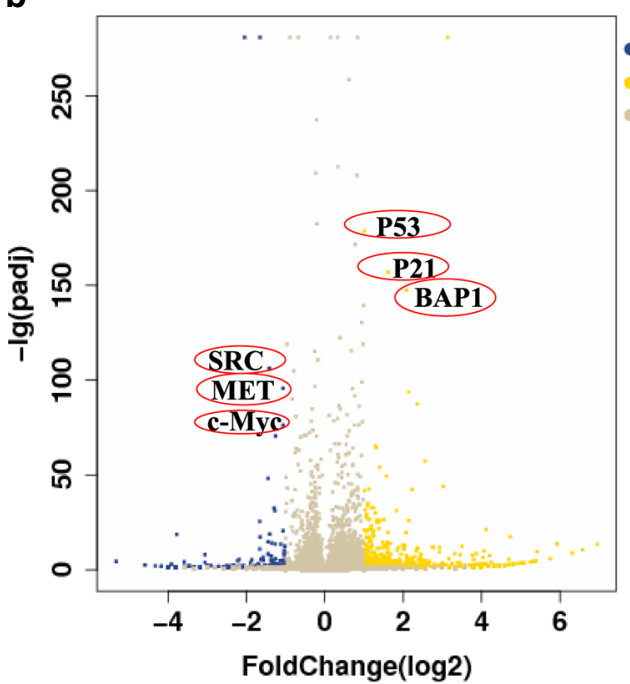

d

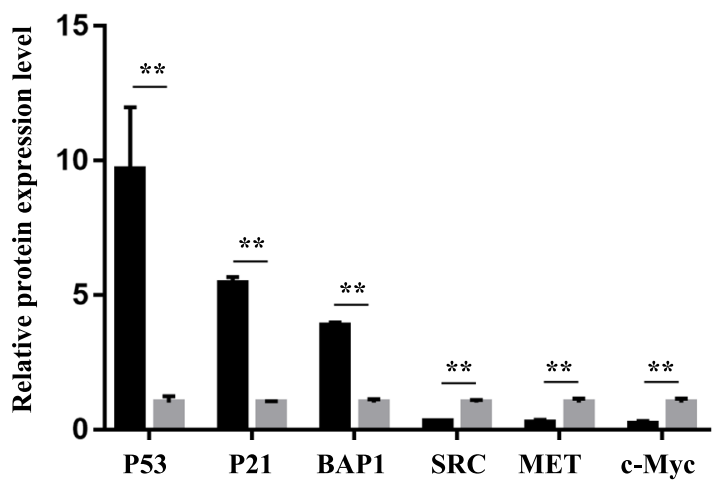

C

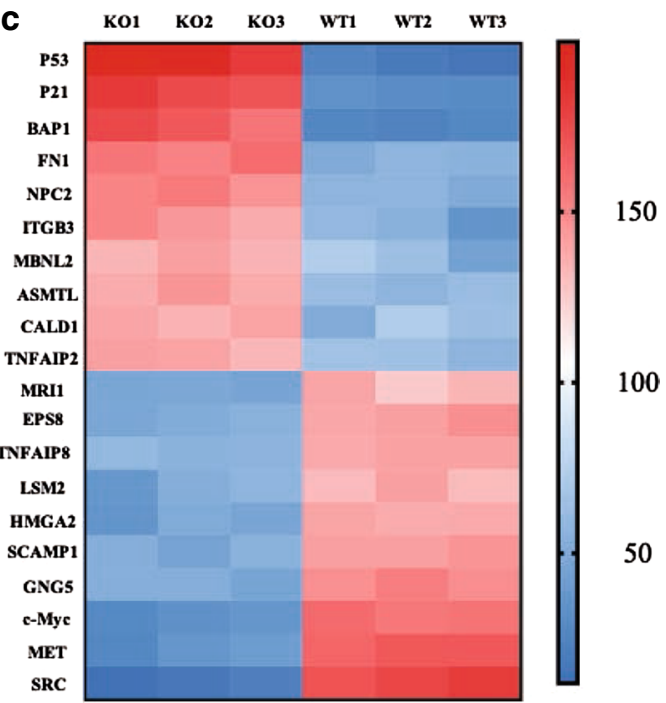

100

50

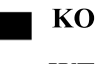

WT

Fig. 4 Construction of TRIM47 knockout cell line of renal cell carcinoma and analysis of mass spectrometry. a Sequencing and Western Blot to detect construction of TRIM47 knockout 769P cells. b, c Volcano map and heat map showing up-regulated differential proteins and down-regulated differential proteins screened out after mass spectrometry. $\mathbf{d}$ The relative protein expression level of different proteins in the two groups of cells (WT and $\mathrm{KO}), \mathrm{P}<0.01$ 
a

769-P cells

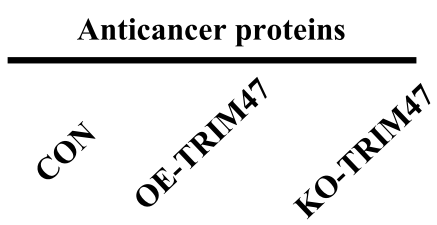

\section{BAP1}

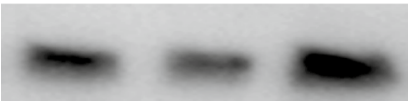

P21

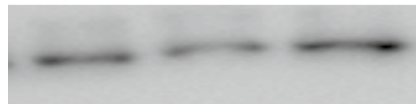

P53

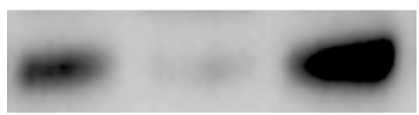

Actin

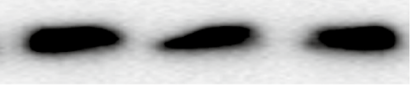

b

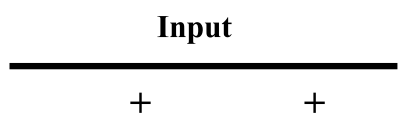

His-P53

Flag-TRIM47

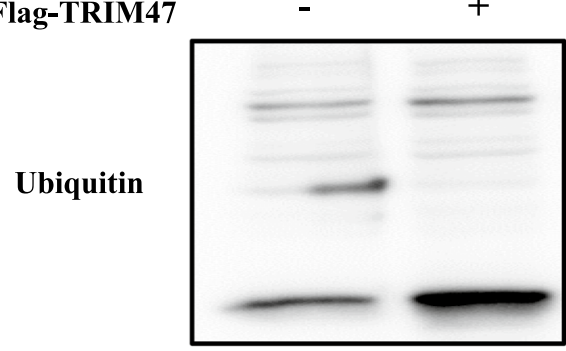

Flag

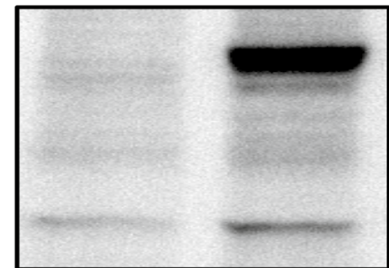

His

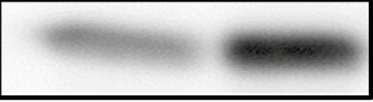

\section{GAPDH}

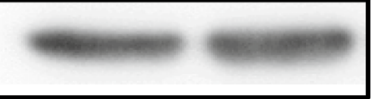

Oncoproteins

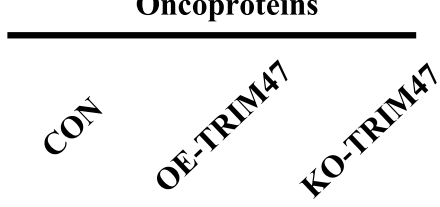

SRC

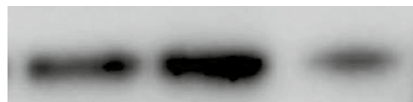

MET

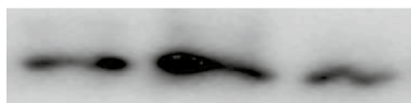

c-Myc

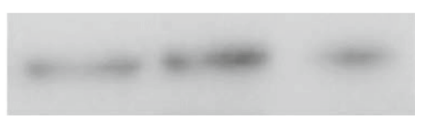

Actin

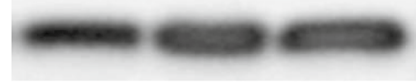

His-P53

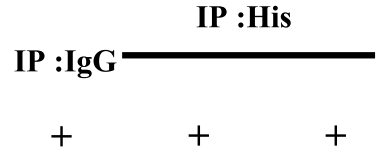

Flag-TRIM47 $+\quad$ - $\quad+$

Ubiquitin

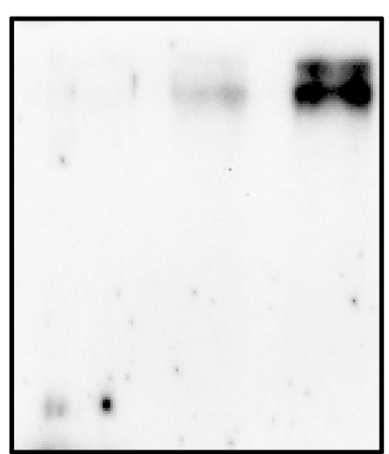

Flag

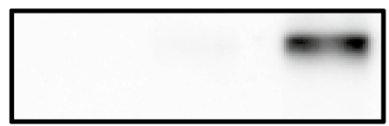

His

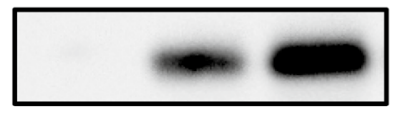

Fig. 5 TRIM47 regulates tumor suppressor protein and oncoprotein and mediate the ubiquitination and degradation of P53 in RCC. a Detection of the expression of anticancer proteins and oncoproteins after TRIM47 overexpression or knockout in 769P cell line. b The stable His-P53 overexpression stable transfected cells and the His-P53 + Flag-TRIM47 overexpression stable transfected cells were constructed and detected by Western blot (left panel). Co-immunoprecipitation was used to detected the interaction of TRIM47 and P53 in the 769P cell line (right panel) 
Flag-TRIM47, and ubiquitination is enhanced. These results showed that TRIM47 and P53 interacted with each other in RCC and the high expression of TRIM47 enhanced P53 ubiquitination (Fig. 5b right panel), suggesting that TRIM47 mediated the ubiquitination and degradation of P53 in RCC.

\section{TRIM47 knockout suppresses tumorigenicity of RCC cells} in nude mice

To determine the effect of TRIM47 expression on RCC tumorigenesis in vivo, 16 nude mice were equally randomized to a KO-TRIM47-769P group and wild-type (WT)-769P group as control. After 3 weeks of the tumor-bearing experiment in mice, the tumor proliferation ability in KO-TRIM47-769P group was reduced significantly as compared with that in WT-769P group (Fig. 6a). Moreover, the tumor growth was significantly slower in mice injected with TRIM47 knockout cells and the weight of the tumor showed significantly difference between the KO-TRIM47-769P group and WT-769P group (Fig. 6b) $(\mathrm{P}<0.01)$.

\section{Discussion}

Members of the TRIM protein family are known to be involved in many biological processes, and changes in abundance or activity are related to a variety of pathological conditions, including viral infections, developmental and neurodegenerative diseases, and cancer occurrence [16]. Recent studies have shown that TRIM proteins positively and negatively regulate carcinogenesis $[17,18]$, and some TRIM proteins can translocate oncogenic chromosomes, where the RBCC motif is fused with another gene, and the possibility is that the RBCC motif induces translocation. It was reported that several of these proteins participated in controlling canceration by regulating the activity of P53 tumor suppressor proteins $[19,20]$. Based on the currently emerging clinical evidence, ubiquitination-mediated degradation of oncogene products or tumor suppressors may be related to cancer occurrence [16]. Among the components of the ubiquitin-protein

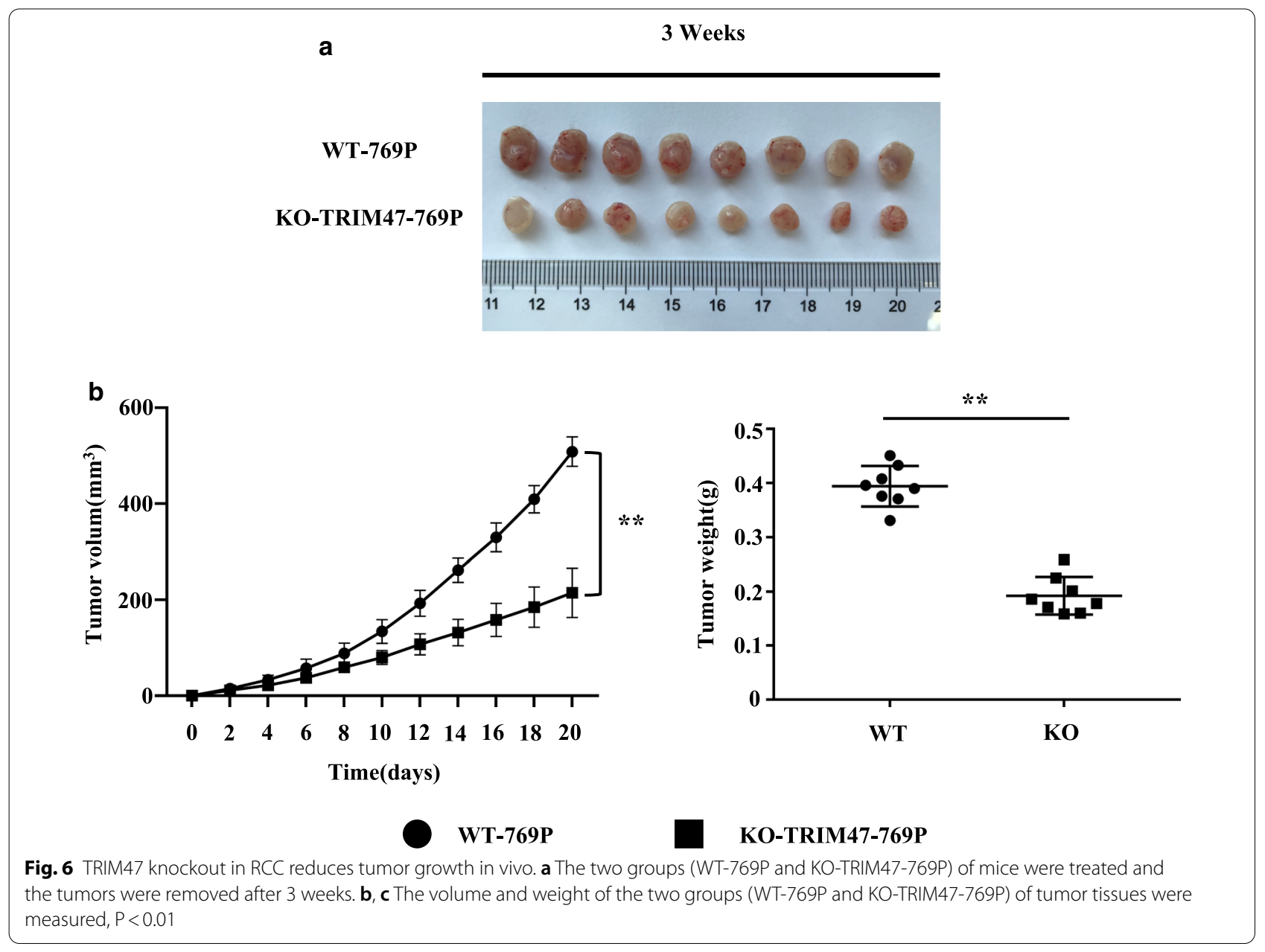


(UPS) system, E3 ubiquitin ligase, which recognizes specific substrates, is considered to be the best diagnostic and therapeutic target for cancer [21]. Most members of the TRIM protein family are endowed with E3 ligase activity due to their RING structure [22-24]. TRIM47 has a RING structure and has been shown to play a role of ubiquitination in colorectal cancer as an E3 ligase, thereby promoting tumor occurrence and metastasis [15].

IHC analysis of the RCC and matched adjacent tissue chips showed that the expression of TRIM47 was up-regulated in RCC tissues. Analysis of TCGA data showed that the expression level of TRIM47 was related to the clinical prognosis of RCC patients, and OS of the patients with high expression was lower than that in the patients with low expression. After overexpression of TRIM47, the proliferation and invasion abilities of 786-O and ACHN RCC cells were significantly enhanced. On the contrary, TRIM47 knockdown mediated by lentiviral shRNA decreased the proliferation and invasion abilities of 769-P and A498 RCC cells significantly. The results of the animal model showed that the use of Crispr/cas9 technology to knock out TRIM47 reduced the tumorbearing ability of nude mice. The above evidence shows that TRIM47 can promote the malignant formation of RCC, suggesting that it may prove to be a potential biomarker for RCC. We then used MS to compare WT-769P with KO-TRIM47-769P. The results showed that there were many significantly differentially expressed proteins between the two types of kidney cancer cell lines, based on which, we selected 6 protein molecules with significant differences, including P53, P21, BAP1 common tumor suppressor proteins, SRC, MET and c-Myc common oncoproteins. Subsequent Western Blot assay showed showed that the protein levels of P53, P21, and BAP1 were significantly down-regulated in RCC 769-P cells after TRIM47 overexpression, and vise versa after TRIM47 knockout. At the same time, the protein levels of SRC, MET and c-Myc were increased after TRIM47 overexpression, and increased after TRIM47 knockout.

The p53 gene is the most common human tumor suppressor gene. It is located on the human chromosome $17 \mathrm{p} 13.1$. As its protein band is located at $53 \mathrm{KDa}$, it is named P53 protein or TP53 protein. P53 protein has the ability to induce cell cycle arrest, senescence and / or apoptosis, which is the core of its anti-tumor mechanism $[25,26]$. The P53 protein has complex functions. Studies have shown that it can specifically bind to DNA, and its activity is regulated by post-translational modifications including phosphorylation, acetylation, and ubiquitination [27-29]. Murine double minute 2 (Mdm2) belongs to an E3 ligase [30], which can interact with P53 itself, and regulate $\mathrm{P} 53$ by inhibiting its transcriptional activity, controlling its subcellular localization and regulating its protein stability [31]. Mdm2 has been shown to exert its E3 ligase function in tumors by directly binding to P53 to mediate its ubiquitination degradation, thereby promoting tumorigenesis and malignant proliferation [32-34]. Similarly, TRIM47 also has E3 ligase activity, and this study showed that TRIM47 could affect P53 protein in RCC, and the results of immunoprecipitation experiments also demonstrated that TRIM47 could interact with P53 protein in RCC, and high TRIM47 expression could enhance ubiquitination of $\mathrm{P} 53$ protein. These results suggest that TRIM47 could promote the generation of malignant biological behavior of RCC by mediating the ubiquitination degradation of P53. Studies showed that the expression of P21 protein was affected by the expression of P53 protein, and the expression of P53 protein in normal cells could promote its expression. In addition, P21 protein was closely related to the cell cycle. As a cyclin-dependent protein kinase inhibitor, it inhibited cyclin (the combination of Cyclin D1), and cyclin-dependent protein kinases CDK4 and CDK6 could block the cell cycle in the G0/G1 phase, and then spared time for DNA damage repair [35-38]. Therefore, the coregulatory role of P53 and P21 in the cell cycle is the basis for affecting tumor suppression. It was found that BAP1 protein could act as a suppressor protein and played a double inhibitory role in RCC [39]. BAP1 protein is also a deubiquitinating enzyme that binds to breast cancer type 1 susceptibility protein (BRCA1) and BRCA1-related RING domain protein 1 (BARD1), which can inhibit their ability to mediate ubiquitination and autoubiquitination, thereby inhibiting tumors [40]. These findings, together with the results of our research, suggest that TRIM47 with ubiquitinase activity could promote the progression of RCC, probably by interacting with BAP1. Protooncoprotein c-Myc promotes the formation of RCC [41]. The increase in SRC protein in renal cancer is related to the poor survival prognosis of patients [42]. MET protein also plays an important role in the pathogenesis of renal cancer by regulating tumor growth, metastasis and angiogenesis [43]. Studies have shown that GAS6/AXL signal can regulate the invasion and metastasis of RCC through the lateral activation of MET by SRC [44]. According to our experimental results, the protein expression level of TRIM47 in renal cell carcinoma is parallel to these three renal cancer-promoting proteins (SRC, MET, c-Myc), and the higher the protein expression level of TRIM47, the stronger the invasion ability of RCC cells, which in turn promotes tumor metastasis. However, TRIM47 and its specific action mechanism have not been defined and need to be further studied. 


\section{Conclusions}

In summary, TRIM47 plays a post-translational modification role in RCC by exerting an E3 ligase activity, mediating ubiquitination and degradation of P53 tumor suppressor protein and promoting malignant progression of RCC cells at the protein level. The role of TRIM47 as a tumor-promoting molecule in promoting the malignant biological behavior of RCC is crucial. However, the specific action mechanism of TRIM47 in RCC needs further exploration. TRIM47 overexpression may prove to be a useful prognostic factor and a potential treatment target for RCC.

\author{
Abbreviations \\ RCC: Renal cell carcinoma; TRIM47: Tripartite motif 47; TKIs: Tyrosine kinase \\ inhibitors; VEGFR: Vascular endothelial growth factor receptors; RING: Really \\ interesting new gene.
}

\section{Acknowledgements}

The project is supported by the laboratory of Translational Medicine Center, Second Military Medical University.

\section{Authors' contributions \\ XC, XP and WZ conceived and designed the study. JC, DX, JC, and LZ per- formed the experiments. JC and DX wrote the manuscript. JC, ZH, YT and CC reviewed and edited the manuscript. All authors read and approved the manuscript.}

\section{Funding}

This study was sponsored by the National Natural Science Foundation of China (No. 81772747, 81974391, 82072806); the Program of Shanghai Academic/Technology Research Leader (No. 19XD1405100); the Clinical Research Plan of SHDC (SHDC2020CR4025); the Shanghai "Rising Stars of Medical Talent" Youth Development Program: Outstanding Youth Medical Talents (X. Cui) and Youth Medical Talents - Specialist Program (X. Pan); the Top-level Clinical Discipline Project of Shanghai Pudong (PWYgf2018-03); the Shanghai Key Medical Specialties Project (ZK2019A09); the Shanghai Municipal Commission of Health and Family Planning (20204Y0042) and the Technology Project of Jiading District Health System (2019-QN-03).

\section{Availability of supporting data}

The data sets supporting the results of this article are included within the article and its supplementary files.

\section{Ethical approval and consent to participate}

The experiment was approved by the ethical committee of the Third Affiliated Hospital of the Second Military Medical University.All the patients gave informed consent and agreed to participate in the study.

\section{Consent for publication}

Written informed consent for publication was obtained from all the participants.

\section{Competing interests}

The authors declare that they have no competing interests.

\section{Author details}

${ }^{1}$ Department of Urology, Third Affiliated Hospital of the Second Military Medical University, 200433, China, Shanghai. ${ }^{2}$ Department of Urology, Changzhou Second People's Hospital, Changzhou 213000, China. ${ }^{3}$ Nanjing University of Traditional Chinese Medicine School of Medical and Life Sciences, Nanjing 210023, China.

Received: 21 November 2020 Accepted: 10 February 2021 Published online: 23 February 2021
References

1. Bray F, Ferlay J, Soerjomataram I, et al. Global cancer statistics 2018: GLOBOCAN estimates of incidence and mortality worldwide for 36 cancers in 185 countries. CA Cancer J Clin. 2018;68(6):394-424.

2. Kenney PA, Wood CG. Integration of surgery and systemic therapy for renal cell carcinoma. Urol Clin North Am. 2012;39(2):211-31.

3. Fisher R, Gore M, Larkin J. Current and future systemic treatments for renal cell carcinoma. Semin Cancer Biol. 2013;23(1):38-45.

4. Choueiri TK, Motzer RJ. Systemic therapy for metastatic renal-cell carcinoma. N Engl J Med. 2017;376(4):354-66.

5. Barata PC, Rini BI. Treatment of renal cell carcinoma: current status and future directions. CA Cancer J Clin. 2017;67(6):507-24.

6. Motzer RJ, Hutson TE, Tomczak $P$, et al. Sunitinib versus interferon alfa in metastatic renal-cell carcinoma. N Engl J Med. 2007;356(2):115-24.

7. Ficarra V, Novara G. Kidney cancer: Characterizing late recurrence of renal cell carcinoma. Nat Rev Urol. 2013;10(12):687-9.

8. Gack MU, Shin YC, Joo CH, et al. TRIM25 RING-finger E3 ubiquitin ligase is essential for RIG-I-mediated antiviral activity. Nature. 2007;:446(7138):916-20.

9. Allton K, Jain AK, Herz HM, et al. Trim24 targets endogenous p53 for degradation. Proc Natl Acad Sci USA. 2009;106(28):11612-6.

10. Song H, Liu B, Huai W, et al. The E3 ubiquitin ligase TRIM31 attenuates NLRP3 inflammasome activation by promoting proteasomal degradation of NLRP3. Nat Commun. 2016;7:13727.

11. Ji YX, Huang $Z$, Yang $X$, et al. The deubiquitinating enzyme cylindromatosis mitigates nonalcoholic steatohepatitis. Nat Med. 2018;24(2):213-23.

12. Vandeputte DA, Meije CB, van Dartel M, et al. GOA, a novel gene encoding a ring finger B-box coiled-coil protein, is overexpressed in astrocytoma. Biochem Biophys Res Commun. 2001;286(3):574-9.

13. Fujimura $T$, Inoue $S$, Urano $T$, et al. Increased expression of tripartite motif (TRIM) 47 is a negative prognostic predictor in human prostate cancer. Clin Genitourin Cancer. 2016;14(4):298-303.

14. Han $Y$, Tian $H$, Chen $P$, et al. TRIM47 overexpression is a poor prognostic factor and contributes to carcinogenesis in non-small cell lung carcinoma. Oncotarget. 2017;8(14):22730.

15. Liang Q, Tang C, Tang M, Zhang Q, Gao Y, Ge Z. TRIM47 is up-regulated in colorectal cancer, promoting ubiquitination and degradation of SMAD4. J Exp Clin Cancer Res. 2019;38(1):159.

16. Valeria Cambiaghi V, Giuliani S, Lombardi, et al. TRIM proteins in cancer. Adv Exp Med Biol. 2012;770(770):77-91.

17. Chen Yu, Guo Y, Yang H, et al. TRIM66 overexpresssion contributes to osteosarcoma carcinogenesis and indicates poor survival outcome. Oncotarget. 2015;6(27):23708-19.

18. Joo HM, Kim JY, Jeong JB, et al. Ret finger protein 2 enhances ionizing radiation-induced apoptosis via degradation of AKT and MDM2. Eur J Cell Biol. 2011;90(5):420-31.

19. Caratozzolo MF, Valletti A, Gigante M, et al. TRIM8 anti-proliferative action against chemo-resistant renal cell carcinoma. Oncotarget. 2014;5(17):7446-57.

20. Elabd S, Meroni G, Blattner C. TRIMming p53's anticancer activity. Oncogene. 2016;35(43):5577-84

21. Nakayama KI, Nakayama K. Ubiquitin ligases: cell-cycle control and cancer. Nat Rev Cancer. 2006;6(5):369-81.

22. Reddy BA, van der Knaap JA, Bot AG, et al. Nucleotide biosynthetic enzyme GMP synthase is a TRIM21-controlled relay of p53 stabilization. Mol Cell. 2014;53(3):458-70.

23. Liu B, Zhang M, Chu H, et al. The ubiquitin E3 ligase TRIM31 promotes aggregation and activation of the signaling adaptor MAVS through Lys63-linked polyubiquitination. Nat Immunol. 2017;18(2):214-24.

24. Liu J, Zhang C, Wang XL, et al. E3 ubiquitin ligase TRIM32 negatively regulates tumor suppressor p53 to promote tumorigenesis. Cell Death Differ. 2014;21(11):1792-804.

25. Levine AJ. p53, the cellular gatekeeper for growth and division. Cell. 1997:88(3):323-31.

26. Vousden KH, Lu X. Live or let die: the cell's response to p53. Nat Rev Cancer. 2002;2(8):594-604.

27. Canman C, Lim D, Cimprich K, et al. Activation of the ATM kinase by ionizing radiation and phosphorylation of $\mathrm{p} 53$. Science. 1998;281(5383):1677-1679.

28. Brooks CL, Gu W. The impact of acetylation and deacetylation on the p53 pathway. Protein Cell. 2011;2(6):456. 
29. Chan WM. Ubiquitination of p53 at multiple sites in the DNA-binding domain. Mol Cancer Res. 2006:4(1):15-25.

30. Kubbutat MHG, Jones SN, Vousden $\mathrm{KH}$. Regulation of p53 stability by Mdm2. Nature. 1997;387(6630):299-303.

31. Boehme KA, Blattner C. Regulation of p53-insights into a complex process. Crit Rev Biochem Mol Biol. 2009;44(6):367-92.

32. Ganguli G, Wasylyk B. p53-independent functions of MDM2. Mol Cancer Res. 2003 Dec;1(14):1027-35.

33. Vargas DA, Takahashi S, Ronai Z. Mdm2: a regulator of cell growth and death. Adv Cancer Res. 2003;89:1.

34. Bouska A, Lushnikova T, Plaza S, Eischen CM. Mdm2 promotes genetic instability and transformation independent of p53. Mol Cell Biol. 2008;28(15):4862-74.

35. Schafer KA. The cell cycle: a review. Vet Pathol. 1998;35(6):461-78.

36. Camacho-Urkaray E, Santos-Juanes J, Gutiérrez-Corres FB, et al. Establishing cut-off points with clinical relevance for bcl-2, cyclin D1, p16, p21, p27, p53, Sox11 and WT1 expression in glioblastoma - a short report. Cell Oncol. 2018;41(2):213-21.

37. He G, Siddik ZH, Huang Z, et al. Induction of p21 by p53 following DNA damage inhibits both Cdk4 and Cdk2 activities. Oncogene. 2005;24(18):2929-43.

38. Jun GJ, Zhong GG, Ming ZS. miR-218 inhibits the proliferation of glioma U87 cells through the inactivation of the CDK6/cyclin D1/p21Cip1/Waf1 pathway. Oncol Lett. 2015;9(6):2743-9.
39. Piva F, Santoni M, Matrana MR, et al. BAP1, PBRM1 and SETD2 in clear-cell renal cell carcinoma: molecular diagnostics and possible targets for personalized therapies. Expert Rev Mol Diagn. 2015;15(9):1201-10.

40. Nishikawa H, Wu W, Koike A, et al. BRCA1-associated protein 1 interferes with BRCA1/BARD1 RING heterodimer activity. Cancer Res. 2009;69(1):111-9.

41. Bailey ST, Smith AM, Kardos J, et al. MYC activation cooperates with Vhl and Ink4a/Arf loss to induce clear cell renal cell carcinoma. Nat Commun. 2017;8:15770.

42. Suwaki N, Vanhecke E, Atkins KM, et al. A HIF-regulated VHL-PTP1B-SrC signaling axis identifies a therapeutic target in renal cell carcinoma. Sci Transl Med. 2011;3(85):85ra47.

43. Harshman LC, Choueiri TK. Targeting the hepatocyte growth factor/c-Met signaling pathway in renal cell carcinoma. Cancer J. 2013;19(4):316-23.

44. Rankin EB, Fuh KC, Castellini L, et al. Direct regulation of GAS6/AXL signaling by HIF promotes renal metastasis through SRC and MET. Proc Natl Acad Sci USA. 2014;111(37):13373-8.

\section{Publisher's Note}

Springer Nature remains neutral with regard to jurisdictional claims in published maps and institutional affiliations.
Ready to submit your research? Choose BMC and benefit from:

- fast, convenient online submission

- thorough peer review by experienced researchers in your field

- rapid publication on acceptance

- support for research data, including large and complex data types

- gold Open Access which fosters wider collaboration and increased citations

- maximum visibility for your research: over $100 \mathrm{M}$ website views per year

At BMC, research is always in progress.

Learn more biomedcentral.com/submissions 